\title{
correspondence
}

\section{Nutritional landmark}

SIR,-Reports prepared by expert committees on specific areas of nutrition are frequently issued, notably by the Food and Agricultural Organisation and the World Health Organisation. It is, however, an event of significance when a comprehensive survey of food and nutrition research in a single country with a strong tradition of scientific and medical investigation is assembled by a large team representative of the available experts. It is indeed a considerable achievement to have synthesised such a readable report from the documents provided by the various working parties, to one of which I was a minor and temporary contributor. The product is a landmark document, which appears at a time when the bridge between basic research and applied research in the national interest must be strengthened in the interests of survival. Food and nutrition research needs to attract original minds trained in the basic sciences. This document should provide the points of contact. It is thus entirely proper that the committee responsible for assembling the report should have included Sir Douglas Black, Chief Scientist to the Department of Health and Social Security and responsible in Britain for implementing contracts in areas of applied research.

I write this comment because a petulant and partly irrelevant review appeared in Nature (January 10) castigating the report because of insufficient emphasis on social nutrition, and ridiculing the priority areas for research identified in the report. On the contrary, the report correctly identifies and summarises current knowledge in numerous areas of nutritional research that are of great importance to human health and survival: the role of nutrition in vascular disease, diet and cancer, dietary fibre and the spectrum of diseases in a population, the aetiology of osteoporosis; nutritional aspects of renal disease, to name some in only one section of the report. The current problems in food and nutrition research are numerous, important and challenging. Further research into the areas identified in this comprehensive document should allow governments to plan research strategies, to test new food sources and to monitor the population more efficiently. Finally, the present disreputable status of nutrition research among basic scientists and the medical profession will also be materially mitigated by the vigorous well-founded directions indicated in the report.

$$
\text { H. V. MunRo }
$$

Massachusetts Institute of Technology Cambridge, Mass.

\section{Re-use of vials}

SIR,-In the February 27 issue, Professor Born rightly drew attention to the need to minimise waste in laboratories and cited as a specific example the destruction of scintillation vials after being used only once. It may be of general interest, therefore, that in this department we have for a number of years successfully re-used vials without risking contamination or any of the other problems which Professor Born mentioned. The procedure we have adopted involves placing the radioactive sample and scintillation fluid in an ordinary $5 \mathrm{~cm} \times 1.2 \mathrm{~cm}$ diameter specimen tube that is inserted into the vial for counting. This simple method has the following advantages: (1) the vials themselves are not contaminated; (2) the volume of scintillation fluid is only $2 \mathrm{ml}$, thus reducing costs; (3) the specimen tubes are very much cheaper than the scintillation vials. They may be either discarded or used for other purposes after normal cleaning.

The method works particularly well for non-aqueous samples-for example, materials on membrane filters or papers - since in these cases there is no reduction in either the counting efficiency or the amount of labelled material used in the assay.

Department of Biochemistry,

King's College, London

\section{Science and technology}

SIR,--In his paper on the changing relationship between science and technology (August 23, 1974) Langrish quotes evidence from previous citation studies which, he asserts, support his views that "British university research has little impact on British industry". We wish to challenge the view that generalisations can be made across all fields of scientific endeavour on the usefulness or otherwise of university research to industry on the basis of the evidence provided by citation analysis.

On the assumption that industrial reviewers will cite any university research which they consider to be of relevance to industrial activity, Langrish chose seven review articles written by British industrial chemists in the 1967 volume of Reports on the Progress of Applied Chemistry, and found that the papers which the reviewers considered most relevant to technological developments in the seven areas were largely of non-university origin. The areas chosen were: phenolic resins, microbiological techniques in manufacture, water treatment, plastics, polyolefins, synthetic detergents and poultry and eggs. In only one area (microbiological techniques) was the influence of university research dominant, and this indicated to the author the importance of the universities in generating new techniques for industrial uptake.

We suggest, however, that a citation study such as this fails to take into account the fact that it is much more likely that there will be university research activity in the area of microbiological techniques, for example, than in water treatment, and that there may be certain major areas of scientific enquiry in which the institutional sources of citations are quite different from the sources found in the disparate subject-areas used as samples by Langrish. In particular, we suggest that the conclusions reached in citation studies of the physical sciences do not have general validity and, in order to demonstrate this, we carried out a study of the institutional sources of citations in five biologically oriented review articles in Reports on the Progress of Applied Chemistry. All the articles were concerned with well established areas of scientific enquiry, and showed that in terms of citations, the universities outstripped every other sector.

One should be wary of generalisations about the industrial utility of university research which rely on evidence from citation data. Langrish has stressed the implications of his citation data for policy makers in government and industry, and the fact that we have been able to obtain results which differ so widely from his raises serious doubts as to the validity of the technique of citation analysis in the determination of the relationship between science and technology. Policy decisions cannot and should not be made on the basis of such dubious evidence. 